\title{
Spice drugs: los cannabinoides como nuevas drogas de diseño
}

\section{Spice drugs: cannabinoids as a new designer drugs}

\author{
Cristina Mustata ${ }^{1,2}$; Marta Torrens ${ }^{1,3}$; \\ Ricardo Pardo ${ }^{1,2}$; Clara Pérez ${ }^{1}$, 2; The Psychonaut \\ WEB MAPPING GROUP*; MAGI FARRÉ $E^{1,2}$
}

Unidad de Farmacología Humana y Neurociencias, Programa de Neuropsicofarmacología, IMIM-Hospital del Mar, Barcelona Universidad Autónoma de Barcelona

3 Unidad de Toxicomanías, Institut d'Atenció Psiquiàtrica, Salut Mental i Toxicomanies, IMIM-Hospital del Mar, Barcelona

* Fabrizio Schifano, Paolo Deluca, Zoe Davey, Ornella Corazza, Marta Torrens, Magi Farre, Arvid Skutle, Liv Flesland, Lucia di Furia, Stefania Pagani, Valentina Minelli, Miia Mannonen, Teuvo Peltoniemi, Aino Majava, Norbert Scherbaum, Holger Siemann, Peer van der Kreeft.

Enviar correspondencia a:

Magi Farré. Unidad de Farmacología Humana y Neurociencias,

IMIM-Hospital del Mar, calle del Doctor Aiguader 88,

08003 Barcelona.

E-mail: mfarre@imim.es

\section{RESUMEN}

Desde 2004 se venden en Internet y tiendas especializadas (smart shops) algunos preparados conteniendo mezclas de hierbas para fumar con la marca de Spice drugs. Estos productos se publicitan como una mezcla exótica de incienso aromático y se especifica que no son aptos para uso humano. Muchos usuarios, tras consumir estos productos fumados, refieren que sus efectos son similares a los del cannabis. Los preparados Spice drugs se han hecho muy populares en los foros de Internet sobre drogas ya que podrian representar la posibilidad de obtener un sustituto legal del cannabis. Los análisis forenses de algunos de estos preparados encontraron la presencia de agonistas cannabinoides sintéticos muy potentes como el JWH-018, CP 47497, JWH-073 y HU-210. Hay muy pocos datos sobre sus propiedades farmacológicas en animales y ninguno sobre su toxicidad. En la actualidad, no se sabe casi nada sobre su farmacología, toxicología y seguridad en humanos, si exceptuamos las opiniones de los consumidores en Internet. Ni los ingredientes vegetales, ni ninguno de los cannabinoides encontrados se hallan en las listas de sustancias controladas de las convenciones de 1961 o 1971 de la ONU. Recientemente, algunos paises han emprendido acciones legales para prohibir o controlar los preparados Spice drugs y similares. Estos cannabinoides pueden considerarse nuevas sustancias a añadir a la lista de drogas de diseño.

Palabras clave: Spice drugs, cannabinoides sintéticos, drogas de diseño, JWH-018, CP 47497, JWH-073, HU-210.

\section{ABSTRACT}

Some smokable herbal mixtures under the brand name Spice drugs have been sold on the Internet and in specialised shops (smart shops) since 2004. The mixtures are advertised as an exotic incense blend which releases a rich aroma and not for human consumption. When smoked, Spice drugs products have been reported by some users to have effects similar to those of cannabis. Spice drugs have received intensive attention in drug forums due to the possibility to obtain a non legal substitute of cannabis. Forensic analyses have found different potent synthetic cannabinoid agonists in some Spice drugs products, as JWH018, CP 47497, JWH-073 and HU-210. There are few data about its pharmacological properties in animals, but nothing about its toxicity. At present, almost nothing is known about the pharmacology, toxicology and safety profile of such compounds in humans, except the opinions of consumers in internet forums. Neither the herbal ingredients of Spice drugs, nor any of the synthetic cannabinoids found in them, are internationally controlled under the 1961 or 1971 UN drug control conventions. Some European countries have recently taken legal actions to ban or otherwise control Spice drugs products and related compounds. These cannabinoid substances can be considered as new products to be added to the list of "designer drugs".

Key words: Spice drugs, synthetic cannabinoids designer drugs, JWH-018, CP 47497, JWH-073, HU-210. 
Se denominan "drogas de diseño" (designer drugs) o "drogas de síntesis" a un grupo heterogéneo de sustancias psicoactivas de origen sintético, creadas a partir de la modificación de la estructura química de algunos productos naturales 0 de sustancias utilizadas como medicamentos. Se acuñó este término para definir nuevas drogas, que eran sintetizadas en laboratorios clandestinos y que no se encontraban en las listas internacionales de sustancias prohibidas. Aunque es cierto que se sintetizaron productos nuevos, lo más común fue redescubrir sustancias conocidas pero olvidadas por diferentes motivos. En la actualidad, los laboratorios clandestinos producen sustancias ilegales y de tanto en tanto, aparecen productos novedosos que, al ser descubiertos, pasan a incluirse poco después en las mencionadas listas. Un aspecto importante es que muchas de ellas pueden llegar al consumidor sin haber pasado un cribado fármaco-toxicológico para conocer sus efectos y/o toxicidad en animales y humanos. Muchas de estas sustancias, especialmente, antes de prohibirse, se pueden adquirir en tiendas especializadas (smart shops) y sobre todo en tiendas online sin demasiados problemas, incluso tras su prohibición ${ }^{1}$

Las principales drogas de diseño son derivados de la fenil-etilamina (similares a la anfetamina y/o la mescalina) y de la triptamina (similares a la dimetiltriptamina)' ${ }^{1}$ Muy recientemente algunos derivados cannabinoides sintéticos se han sumado a las lista de drogas de diseño al encontrarse entre los componentes de las Ilamadas Spice drugs.

\section{SPICE DRUGS}

Desde 2004 se han ido vendiendo, sobre todo en páginas de Internet, unos sobres conteniendo una mezcla de hierbas exóticas y extractos de plantas aromáticas con el nombre de Spice. Entre ellos podemos mencionar Spice Silver, Spice Gold, Spice Diamond, Spice Arctic Synergy, Spice Tropical Synergy y Spice Egypt. Además, hay muchos otros preparados a base de plantas que se dice que tienen una composición similar a los productos Spice como por ejemplo Yucatan Fire, Smoke, Sence, ChillX, Highdi's Almdröhner, Earth Impact, Gorillaz, Skunk, Genie, Galaxy Gold, Space Truckin, Solar Flare, Moon Rocks, Blue Lotus, Aroma, Scope, entre otros.

Estos productos se comercializaron como similares al incienso para utilizar durante sesiones de aromaterapia, yoga o meditación. Se vendían bajo mensajes como "una mezcla de incienso que libera un aroma" y siempre advirtiendo "no apto para el uso humano"2. En su etiqueta se indica que el producto contiene entre 0,4-3 g de diferentes especies vegetales. Entre ellas se encuentran: Canavalia maritima, Nymphaea alba, N. Caerulea, Scutellaria nana, Pedicularis densiflora, Leonotis leonuru, Zornia latifolia o Zornia diphyIla, Nelumbo nucifera, Leonurus sibiricus, Althaea officinalis, Rosa canina. Estas plantas parecen no haber sido elegidas al azar, ya que algunas de ellas han sido tradicionalmente reconocidas como sustitutos de la marihuana, como es el caso de la Pediculares densiflora ("indio guerrero") y Leonotis leonorus ("la cola de león"), que se sabe que pueden producir efectos psicoactivos ${ }^{2,3}$.
El papel de Internet en el fenómeno Spice drugs ha sido muy importante, tanto por la publicidad de las tiendas online como por las opiniones de los consumidores en foros especializados en drogas. Así, el consumo fue incrementándose al descubrirse que se podian fumar y que algunos provocaban efectos similares al cannabis. Según los comentarios vertidos por los propios consumidores en los foros de Internet, estas sustancias podrían ser el sustituto legal del cannabis. Además, tras su consumo, los tests habituales de detección de los metabolitos del delta-9-tetrahidrocannabinol (THC) en orina resultan negativos ${ }^{2}$.

Los patrones de consumo se han restablecido siguiendo las declaraciones y experiencias de los propios usuarios a través de los foros de Internet. La conclusión fue que las Spice se pueden fumar, algunas veces junto con el cannabis, inhalar y otras veces consumir como una infusión ${ }^{2}$. En cuanto a la dosis consumida diariamente o la relación dosisefecto, hasta la fecha, la información disponible en este ámbito es muy limitada, explicable por el hecho de que los productos Spice acaban de llegar al mercado de las drogas. Algunos usuarios explican unos efectos subjetivos de intensidad variable, que son muy similares a los de cannabis y se inician muy rápido, o incluso pueden ser algo diferentes y de larga duración. En algunos países (Alemania, Italia) los servicios de urgencias médicas se han enfrentado con casos de intoxicación por algún tipo de producto Spice. Los efectos más llamativos fueron taquicardia, agitación psicomotora, disminución y del nivel de conciencia que se recuperó rápidamente. En ningún caso hubo confirmación toxicológica de drogas en sangre $u$ orina².

Las primeras informaciones oficiales sobre Spice drugs fueron los resultados de las investigaciones alemanas y austriacas que identificaron en algunos productos Spice sustancias con propiedades psicoactivas. A finales de 2008, estos gobiernos notifican oficialmente al European Monitoring Centre for Drugs and Drug Addiction (EMCDDA) los resultados del análisis realizado por THC-Pharm y AGES PharmMED, en los que encontraron el compuesto JWH-018 (1-pentil-3(1-naftoil)indol) en los sobres de algunas Spice ${ }^{2,4}$. Lo que se sabia hasta entonces sobre JWH-018 provenía de las escasas publicaciones en revistas de farmacología experimental y de química médica, en las que se describe como un agonista para los receptores cannabinoides CB1 y CB2. En animales sus efectos eran incluso más intensos que los del THC. Poco más tarde se identificaron otros compuestos en preparados Spice: el CP 47497 (5-(1,1-dimetilheptil)-2-[(1R,3S)-3hidroxiciclohexil]-fenol), el homologo C8 del CP 47497 y uno de sus estereoisómeros y la oleamida, todos ellos con actividad cannabinoide ${ }^{2,4,5}$.

Casi al mismo tiempo, en Dinamarca y Países Bajos se identificó otro compuesto cannabinoide de sintesis de la familia JWH, el Ilamado JWH-073, un análogo de tipo alquil del JWH-018².

La investigación de los productos Spice no ha sido sólo un fenómeno europeo. En el primer trimestre de 2009, la Drug Enforcement Administration (DEA) de los EEUU informó sobre el hallazgo de otro cannabinoide sintético mucho más potente, el HU-210 en un pequeño lote de productos Spice 
${ }^{6}$. Un grupo de investigación japonés del National Institute of Health Sciences confirmó la presencia del CP 47497 y su homologo $\mathrm{C} 8$ en algunas muestras de Spice?.

Cabe mencionar que centros asociados a la red europea Reitox en Suecia ya redactaron en 2007 un informe sobre algunos productos marca Spice. A partir de todos los hallazgos mencionados, en 2008, el EMCDDA que coordina el Sistema de Alerta Temprana sobre las nuevas sustancias psicotrópicas (EWS, Early Warning System) inicia una campaña de seguimiento para estos productos. Todo ello finaliza recientemente con la prohibición de estos productos en varios países de la Unión Europea (UE) y la publicación de un informe de un grupo de trabajo sobre las Spice drugs bajo el auspicio del EMCDDA².

\section{CANNABINOIDES SINTÉTICOS}

La farmacología del cannabis, del THC y del sistema endocanabinoide humano se vienen estudiando intensamente desde hace más de 40 años.

De los casi 70 cannabinoides naturales descritos en la planta de cannabis, el THC fue el primero en ser aislado y posteriormente sintetizado. A partir de entonces, se han sintetizado centenares de derivados, tanto con propiedades agonistas como antagonistas, en laboratorios universitarios o de la industria farmacéutica. La finalidad es obtener sustancias con el perfil de efectos del THC pero sin su actividad psicoactiva. Esta investigación ha logrado definir la relación estructura-actividad y la afinidad para los receptores cannabinoides $(\mathrm{CB} 1, \mathrm{CB} 2)^{8}$. Los modelos experimentales en anima- les que pueden predecir la psicoactividad de un cannabinoide han sido clásicamente cuatro: la analgesia, la producción de catalepsia, el efecto hipotérmico y la alteración de la actividad locomotora en ratas. En perros se ha empleado el modelo de ataxia. Para predecir su potencial de abuso se utilizan modelos de preferencia de plaza, de discriminación o de autoadministración ${ }^{9}$.

Las diferencias químicas y de actividad, así como la dificultad para medir los efectos psicoactivos en animales, explicarían por qué de todos los compuestos sintetizados hasta la fecha pocos han llegado a estudiarse en humanos y muchos menos han llegado a utilizarse en terapéutica.

Si clasificamos los cannabinoides por su actividad y estructura química (Figura 1), los agonistas se clasifican en cuatro grupos ${ }^{8,9}$.

1. Cannabinoides clásicos (dibenzopiranos). Presentan la estructura tricíclica característica de los cannabinoides (análoga a la del THC). Los más investigados han sido el propio $\mathrm{THC}$, el $\Delta 8-\mathrm{THC}$, el HU-210 y su enantiómero HU-211 (dexanabinol) y el levonantradol. El HU-210 es un agonista para los receptores $\mathrm{CB} 1$ y $\mathrm{CB} 2$, con una potencia entre 60 y 100 veces mayor que el THC en el modelo de discriminación. Hasta ahora es seguramente el cannabinoide sintético más potente descrito ${ }^{10,11,12}$.

2. Cannabinoides no clásicos. Son análogos bicíclicos o tricíclicos de los cannabinoides clásicos. Incluyen el CP55940 (potente agonista no selectivo), el CP 47497 y sus homólogos. El CP 47497 es un agonista con una afinidad 20 veces mayor para el receptor CB1 que el THC. También ha demostrado mayor potencia que THC como analgésico

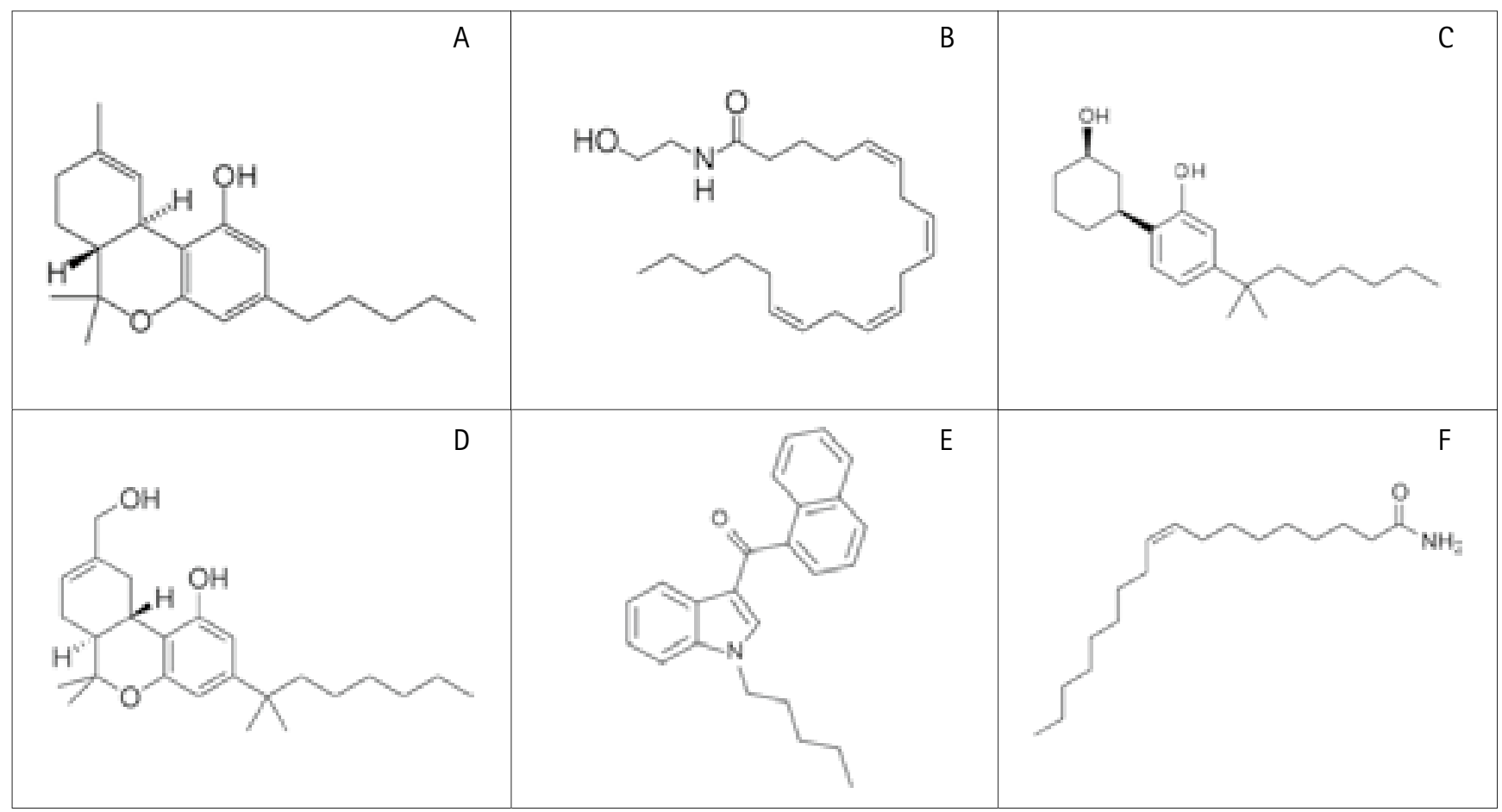

Figura 1. Estructura química de diferentes cannabinoides. A) THC; B) Anandamida; CP 47497; D) HU-210;

E) JWH-018; F) Oleamida 
(6-17 veces), para disminuir la actividad locomotora (5 veces), producir hipotermia (6 veces), pero tiene resultados similares a los de THC en el modelo de discriminación ${ }^{13,14}$

3. Aminoalkilindoles. Tienen una estructura química totalmente diferente a los anteriores, pero con actividad cannabinomimética demostrada. Su representante y cabeza de serie es el WIN 55212-2. Destacan además el JWH-015 y su homologo, el JWH-018 y el JWH-073. Como se ha comentado, el JWH 018, es un agonista con mayor afinidad para los receptores $C B 1$ y CB2 que el THC. En los estudios in vivo en ratas, disminuye la actividad locomotora, produce analgesia, hipotermia y catalepsia similar al THC. El JWH-073 tiene mayor afinidad para el receptor $\mathrm{CB} 1$, pero afinidad similar a la del THC para el receptor $\mathrm{CB} 2$. Tiene la misma potencia que el THC para modificar la actividad locomotora, producir hipotermia y analgesia, pero no se ha estudiado el efecto de catalepsia. No se conocen sus efectos en pruebas de discriminación de drogas ${ }^{15,16}$.

4. Endocanabinoides (eicosanoides). Son productos endógenos que se sintetizan a partir de precursores fosfolipídicos de las membranas celulares. La mayoría son derivados del ácido araquidónico que se unen con mayor o menor afinidad a los receptores cannabinoides. Los más estudiados han sido la araquidoniletanolamida o anandamida, el 2-araquidonilglicerol y la noladina éter. La anandamida es el prototipo de la clase y se muestra como un agonista parcial para los receptores $\mathrm{CB} 1$ y $\mathrm{CB} 2$, con una potencia parecida a la del $\mathrm{THC}^{9,17}$. El 2-araquidonilglicerol es un agonista para los receptores CB1 y CB2, y tiene una mayor afinidad que la de anandamida para los receptores $\mathrm{CB}^{19}{ }^{97}$. La oleamida, que es una amida de ácido oléico, tiene efectos similares a la anandamida sobre los receptores $\mathrm{CB} 1^{18,19}$.

En el caso de los antagonistas cannabinoides, el grupo más estudiado es el de los diarilpirazoles. El pionero del grupo, el SR141716 (rimonabant), exhibe una elevada selectividad por el receptor $\mathrm{CB}^{20}$. El rimonabant es el cannabinoide antagonista que más lejos ha llegado en el desarrollo farmacéutico, ya que estuvo aprobado en la Unión Europea para el tratamiento de la obesidad antes de ser retirado del mercado en 2008 por haber producido alteraciones psiquiatricas ${ }^{21}$. El SR144528, fue el primer antagonista selectivo para el receptor $\mathrm{CB} 2^{20}$

De todos los cannabinoides sintéticos agonistas existentes pocos han llegado a comercializarse. La nabilona (Cesamet $^{\circledR}$ ) que es un derivado del THC y el Dronabinol (Marinol ${ }^{\circledR}$ ) que es THC sintético, están indicados en algunos países para el tratamiento de las náuseas y vómitos secundarios a la quimioterapia antineoplásica. El dronabinol también tiene como indicación el síndrome de anorexia-caquexia en el contexto de cáncer terminal o del SIDA. Cabe destacar que algunos de los agonistas sintéticos (CT-3, el dexanabinol o el WIN 55212-2, entre otros), se han evaluado o están siendo evaluados en humanos para patologias diversas, fundamentalmente neurológicas y en el dolor ${ }^{22}$.

\section{Efectos de los cannabinoides sintéticos de las Spice drugs en humanos}

Debido a que sólo existen estudios en laboratorio (in vitro o con animales de experimentación) es muy difícil extrapolar los resultados a humanos y poder valorar que riesgos implica su uso. Como el tipo y la cantidad de cannabinoides pueden variar considerablemente dentro de un mismo producto Spice, puede especularse la posibilidad de que agonistas potentes para el receptor CB1 JWH-018 o HU-210 podrian causar casos de intoxicación o la posibilidad de sobredosis 3 .

En un reciente articulo publicado por investigadores forenses alemanes se informa que los efectos del consumo de Spice son similares a los del cannabis ${ }^{5}$. Los autores consumieron un cigarrillo que contenía $0,3 \mathrm{~g}$ del producto Spice Diamond. A los 10 minutos notaron los primeros efectos, con enrojecimiento conjuntival, taquicardia, boca seca y alteración del estado de ánimo y de la percepción. Todos ellos similares a los provocados tras el consumo de cannabis o THC, y con una duración de hasta 6 horas. No se han encontrado más trabajos científicos publicados en humanos. Como conclusión, los efectos descritos son similares a los referidos en los foros de Internet por los usuarios de Spice drugs 4 .

Las dificultades para detectar el fenómeno y conocer los principios psicoactivos en los productos Spice pueden tener algunas explicaciones. Por un lado el propio contenido, ya que algunas hierbas pueden tener efectos similares al cannabis por si mismas; los extractos de las mezclas son matrices muy complejas con múltiples principios químicos; la presencia en muchos casos de algunas sustancias no psicoactivas como la vitamina E que podrian enmascarar la presencia de los compuestos activos lipofílicos; la falta de material de referencia analítico para identificar estos cannabinoides, y en caso de tenerlo, sólo se puede determinar un compuesto y no los otros.

Por otro lado las ventas de estos productos se hicieron a través de Internet o en tiendas especializadas, y por eso las Spice durante mucho tiempo no levantaron sospechas de tráfico ilícito de drogas².

El proceso de fabricación de las Spice sigue siendo un enigma. Por un lado se cree que algunas se elaboran en China, pero no se sabe quién, dónde ni cómo se mezclan las plantas naturales con los cannabinoides sintéticos. Por otro lado, se ha sugerido que los productores de Spice podrian realizar la mezcla al comprar los cannabinoides sintéticos a laboratorios chinos o de otros países donde los sintetizan de forma barata ${ }^{2,4}$. Además, se ha descrito la oleamida como producto contaminante en la fabricación de los envases de plástico ${ }^{23}$. Esta hipótesis se descartaría dado que el precio de las Spice aumenta paralelamente según la cantidad de cannabinoide ofrecida ${ }^{4}$. 


\section{SPICE DRUGS COMO PRODUCTOS CONTROLADOS}

Actualmente ninguno de los productos Spice, ni las plantas que los componen, ni tampoco los cannabinoides sintéticos están considerados a nivel internacional como sustancias ilícitas o controladas en las convenciones de 1961 y 1971 de las Naciones Unidas (ONU).

En el primer trimestre del 2009, en algunos países se hicieron públicas las medidas legales, que no han sido homogéneas. Así, Alemania, Austria y Francia prohiben el tráfico, producción, adquisición y posesión de los productos que contengan compuestos de los grupos JWH y CP, pero en Alemania esta restricción es sólo por un año. En el Reino Unido se exige para el producto Spice Diamond una autorización para comercialización. Hungría prohíbe la distribución de los productos Spice Gold, Spice Diamond y Sence y la de todos los productos que contengan la misma mezcla de hierbas que los anteriores.

A principios de 2009 los representantes EMCDDA y Europol se reunieron y examinaron toda la información disponible hasta entonces y llegaron a la conclusión que por ahora JWH-018, CP 47497 y sus homólogos no cumplen los criterios establecidos por el sistema de alerta temprana, (EWS Operating guidelines) para ser considerados sustancias prohibidas. El caso quedó abierto con la posibilidad de que cualquier información adicional y en cualquier momento podría ser rápidamente investigada².

El fenómeno Spice drugs demuestra de nuevo que existe la posibilidad de introducir en el mercado drogas de diseño evitando durante cierto tiempo los controles fiscalizadores antidroga. Los cannabinoides sintéticos forman parte desde ahora de la lista de las drogas de diseño, aquellas que se sintetizan clandestinamente para obviar los controles impuestos por las listas de sustancias prohibidas o controladas internacionalmente. Lo cierto es que, a fecha de hoy, no sabemos si estamos al principio o al final de este fenómeno con los cannabinoides sintéticos. En todo caso, no sería de extrañar la aparición de fenómenos similares con otros nombres, presentaciones y formas de consumo en el futuro. La caja de Pandora para los cannabinoides sintéticos puede haberse abierto para siempre.

\section{AGRADECIMIENTOS}

Financiado en parte por las ayudas Psychonaut Web Mapping Project (European Commission - EC A/800102 [2006 348]); Red de Trastornos Adictivos (FIS RTARD06/0001/0026); Ministerio de Sanidad - Plan Nacional sobre Drogas (SOC/3386/2004).

\section{REFERENCIAS}

1. Buchanan JF, Brown CR. 'Designer drugs.' A problem in clinical toxicology. Med Toxicol Adverse Drug Exp 1988; 3: 1-17.

2. European Monitoring Centre for Drugs and Drug Addiction. Action on new drugs briefing paper: Understanding the Spice phenomenon (a report from an EMCDDA expert meeting, 6 March 2009, Lisbon) Updated version: 9 June 2009. Disponible en: http://www.emcdda.europa.eu/html.cfm/index78154EN.html [consultado el 02/07/09].

3. Stafford GI, Pedersen ME, Van Staden J, Jäger A. Review on plants with CNS-effects used in traditional South African medicine against mental diseases. J. Ethnopharmacology 2008; 119: 513537.

4. Piggee C. Investigating a not-so-natural high. Anal Chem 2009; 81: 3205-3207.

5. Auwärter $V$, Dresen $S$, Weinmann W, Müller $M$, Pütz M, Ferreirós N. Spice and other herbal blends: harmless incense or cannabinoid designer drugs? Journal of Mass Spectrometry 2009; 44 : 832 -837.

6. Microgram Bulletin, Volume 42, Number 3, March 2009. Disponible en: http://www.usdoj.gov/dea/programs/forensicsci/ microgram/mg0309/mg0309.html. [Consultado el 22/06/09].

7. Uchiyama N, Kikura-Hanajiri R, Kawahara N, Haishima Y, Goda Y. Identification of a Cannabinoid Analog as a New Type of Designer Drug in a Herbal Product. Chem Pharm Bull 2009; 57 : 439-441.

8. Ashton JC, Wright JL, McPartland J.M, Tyndall JDA. Cannabinoid CB1 and CB2 Receptor Ligand Specificity and the Development of CB2-Selective Agonists. Curr Med Chem 2008; 15: 1428-1443.

9. Howlett AC, Barth F, Bonner TI, Cabral G, Casellas P, Devane WA, et al. International Union of Pharmacology. XXVII. Classification of cannabinoid receptors. Pharmacol Rev 2002; 54:161-202.

10. Huffman JW and Sammy G. Duncan SG. Jr. Synthesis and pharmacology of the 1',2'-dimethylheptyl 8-THC: exceptionally potent cannabinoids. Bioorganic \& Medicinal Chemistry Letters 1997; 7: 2799-2804.

11. Järbe TU, Hiltunen AJ, Mechoulam R. Stereospecificity of the discriminative stimulus functions of the dimethylheptyl homologs of 11-hydroxy-delta 8-tetrahydrocannabinol in rats and pigeons. J Pharmacol Exp Ther 1989; 250: 1000-1005.

12. Ottani A and Giuliani D. HU 210: A Potent Tool for Investigations of the Cannabinoid System. CNS Drug Reviews 2001; 7: 131145.

13. Weissman A, Milne GM, Melvin LS Jr. Cannabimimetic Activity from CP-47,497, A Derivative of 3-Phenylcyclohexanol. J Pharmacol Exp Ther 1982; 223: 516-523.

14. Compton DR, Johnson MR, Melvin LS, Martin BR. Pharmacological profile of a series of bicyclic cannabinoid analogs: classification as cannabimimetic agents. J Pharmacol Exp Ther 1992; 260: 201-209.

15. Aung MM, Griffin G, Huffman JW, Wu M, Keel C, Yang B, et al. Influence of the $\mathrm{N}-1$ alkyl chain length of cannabimimetic indoles upon $\mathrm{CB}(1)$ and $\mathrm{CB}(2)$ receptor biding. Drug Alcohol Depend 2000; 60: 133-140.

16. Wiley JL, Compton DR, Dai D, Lainton JA, Phillips M, Huffman $J W$, et al. Structure-Activity Relationships of Indole- and Pyrrole-Derived Cannabinoids. J Pharmacol Exp Ther 1998; 285: 995-1004. 
17. Mechoulam R, Ben-Shabat S. From gan-zi-gun-nu to anandamide and 2-arachidonoylglycerol: the ongoing story of cannabis. Nat Prod Rep 1999; 16: 131-143.

18. Leggett JD, Aspley S, Beckett SR, D'Antona AM, Kendall DA, Kendall DA. Oleamide is a selective endogenous agonist of rat and human CB1 cannabinoid receptors. Br J Pharmacol 2004; 141: 253-262.

19. Mechoulam R, Fride E, Hanus L, Sheskin T. Anandamide may mediate sleep induction. Nature 1997; 389: 25-26.

20. Muccioli GG, Lambert DM. Current knowledge on the antagonists and inverse agonists of cannabinoid receptors. Curr Med Chem 2005; 12: 1361-1394.

21. European Medicines Agency. London, 23 October 2008 Doc. Ref. EMEA/CHMP/537777/2008. Press release. The European Medicines Agency recommends suspension of the marketing authorisation of Acomplia. Disponible en: http://www.emea. europa.eu/humandocs/PDFs/EPAR/acomplia/53777708en.pdf [consultado el 02/07/09].

22. Mohamad Ben Amar. Cannabinoids in medicine: Areview of their therapeutic potencial. J Ethnopharmacol. 2006.; 105: 1-25.

23. Mc Donald GR, Hudson AL, Dun SMJ, You H, Baker GB, Whittal $\mathrm{RM}$, et al. Bioactive Contaminants Leach from Disposable Laboratory Plasticware. Science 2008; 322: 5903-5912. 